\title{
Apoptosis signal-regulating kinase 1 (ASK1) is linked to neural stem cell differentiation after ischemic brain injury
}

Juhyun Song ${ }^{1,4}$, Kyoung Joo Cho ${ }^{1,2,4}$, So Yeong Cheon ${ }^{1,2}$, Sa-Hyun Kim ${ }^{3}$, Kyung Ah Park ${ }^{1}$, Won Taek Lee ${ }^{1}$ and Jong Eun Lee ${ }^{1,2}$

Neural stem cells (NSCs) have been suggested as a groundbreaking solution for stroke patients because they have the potential for self-renewal and differentiation into neurons. The differentiation of NSCs into neurons is integral for increasing the therapeutic efficiency of NSCs during inflammation. Apoptosis signal-regulating kinase 1 (ASK1) is preferentially activated by oxidative stress and inflammation, which is the fundamental pathology of brain damage in stroke. ASK1 may be involved in the early inflammation response after stroke and may be related to the differentiation of NSCs because of the relationship between ASK1 and the p38 mitogen-activated protein kinase pathway. Therefore, we investigated whether ASK1 is linked to the differentiation of NSCs under the context of inflammation. On the basis of the results of a microarray analysis, we performed the following experiments: western blot analysis to confirm ASK1, DCX, MAP2, phospho-p38 expression; fluorescence-activated cell sorting assay to estimate cell death; and immunocytochemistry to visualize and confirm the differentiation of cells in brain tissue. Neurosphere size and cell survival were highly maintained in ASK1-suppressed, lipopolysaccharide (LPS)-treated brains compared with only LPS-treated brains. The number of positive cells for MAP2, a neuronal marker, was lower in the ASK1suppressed group than in the control group. According to our microarray data, phospho-p38 expression was inversely linked to ASK1 suppression, and our immunohistochemistry data showed that slight upregulation of ASK1 by LPS promoted the differentiation of endogenous, neuronal stem cells into neurons, but highly increased ASK1 levels after cerebral ischemic damage led to high levels of cell death. We conclude that ASK1 is regulated in response to the early inflammation phase and regulates the differentiation of NSCs after inflammatory-inducing events, such as ischemic stroke.

Experimental \& Molecular Medicine (2013) 45, e69; doi:10.1038/emm.2013.134; published online 20 December 2013

Keywords: apoptosis signal-regulating kinase 1 (ASK1); brain ischemic injury; neural stem cell; neurogenesis; neuron

\section{INTRODUCTION}

Neurons are highly susceptible to damage; furthermore, dead neuronal cells are not renewable or regenerable. ${ }^{1}$ These neuronal characteristics make it difficult to successfully cure neuronal disease. The activation of intrinsic neural stem cells (NSCs) or the exogenous transplantation of NSC/neural progenitor cells can be applied during neuro-regeneration. ${ }^{2}$ NSCs have been isolated by Reynolds and Weiss ${ }^{3}$ from the adult brain and have the potential for self-renewal and differentiation into neurons, astrocytes and oligodendrocytes. ${ }^{4}$ Endogenous, multipotent progenitor cells are located in the subgranular zone of the hippocampus and the subventricular zone (SVZ), where they proliferate; these cells can migrate to a non-injured area and terminally differentiate into neurons. ${ }^{5}$ Differentiation of these stem cells is orchestrated by various extrinsic and heritable, intrinsic factors that determine the proper spatial and temporal cellular development. ${ }^{6}$ Various stimuli that promote the neurogenesis of NSCs in vivo and in vitro have been identified, for example, retinoic acid, neurotrophins, steroid hormones and insulin-like growth factor $1 .^{7-9}$ The microenvironment that is established by extrinsic and intrinsic factors must be optimal to differentiate NSCs into specific cells in the damaged area.

Apoptosis signal-regulating kinase 1 (ASK1) has been reported to regulate cell fate in many injury conditions and disease models. ASK1 is a mitogen-activated protein kinase

\footnotetext{
${ }^{1}$ Department of Anatomy, Yonsei University, Seoul, Korea; ${ }^{2}$ BK21 Project for Medical Sciences, Yonsei University, College of Medicine, Seoul, Korea and ${ }^{3}$ Department of Clinical Laboratory Science, Semyung University, Chungbuk, Korea

${ }^{4}$ These authors contributed equally to this work.

Correspondence: Professor JE Lee, Department of Anatomy, College of Medicine, Yonsei University, 50 Yonsero Seodaemun-gu, Seoul 120-752, South Korea. E-mail: jelee@yuhs.ac

Received 30 June 2013; revised 4 September 2013; accepted 9 September 2013
} 
kinase kinase (MAPKKK, MAP3K) that is preferentially activated by oxidative stress and inflammation. ASK1 has been linked to the fundamental pathology of brain damage in stroke and has essential roles in various cellular responses, such as apoptosis, cell differentiation and immune responses. ASK1 is a MAP3K that is involved in MAPK signaling pathways and MAPK cascades. ${ }^{10}$ ASK1 activates the p38 MAPK and the c-jun $\mathrm{N}$-terminal-activating kinase pathways ${ }^{11}$ and eventually triggers cell death. Our previous study demonstrated that after cerebral ischemia in the mouse, ASK1 may be associated with neuronal cell death. In addition to being an apoptosis signaling protein, ASK1 has been reported to be a cell fate determinant for NSCs. ${ }^{12}$ In addition, several reports have indicated that ASK1 could promote cellular differentiation and survival rather than apoptosis, although these studies used non-neuronal cells. ${ }^{13,10}$ The findings suggest that ASK1 might have opposing functions that are dependent upon cell type and status.

NSCs and neural progenitor cells have been demonstrated to have a neuroprotective role by regulating inflammatory responses in an injured area. Neurogenesis occurs in the injured area, where newly born neurons migrate into the infarct cortex and provide direct neurotrophic support and cell replacement. ${ }^{14,15}$ Cellular apoptosis occurs during the early, post-stroke inflammation phase. After stroke, modulating the key factor in the early inflammation phase might be useful in clinical therapeutics for treating ischemic stroke.

In this study, we investigated the effect of ASK1 signaling, an early response to inflammation and other injuries, on the survival, proliferation and differentiation of NSCs in inflammatory conditions.

\section{MATERIALS AND METHODS}

\section{Animals}

Male C57BL/6 mice (Orient, GyeongGi-Do, Korea; 8- to 12-week old) were subjected to transient focal cerebral ischemia by intraluminal middle cerebral artery blockade with a nylon suture, as previously described. ${ }^{16}$ After $60 \mathrm{~min}$ of middle cerebral artery occlusion, blood flow was restored by withdrawing the suture, and regional cerebral blood flow was monitored using a laser Doppler flowmeter (Transonic Systems, Inc., Ithaca, NY, USA). All animal procedures and experiments were performed in accordance with the Guide to the Care and Use of Laboratory Animals and were approved by the Association for Assessment and Accreditation of Laboratory Animal Care.

\section{Preparation of the ASK1 targeting siRNA}

A small interfering RNA (siRNA) for ASK1 (Ambion, Austin, TX, USA; sense, 5'-GCUGGUAAUUUAUACACuGtt-3'; antisense, 5'-CAGUGUAUAAAUUACGAGCtt-3'; conc, $5 \mu \mathrm{M}$ ) was prepared to silence ASK1 mRNA. Using an osmotic pump (Alzet, Cupertino, CA, USA), a mixture of siPORT NeoFX (Ambion) and ASK1-siRNA was administered into the cerebral ventricle (mediolateral $1.0 \mathrm{~mm}$; anteroposterior $0.2 \mathrm{~mm}$; dorsoventral $3.1 \mathrm{~mm}$ ) for 3 days before transient focal cerebral ischemia.

For treating siASK1 on NSCs, siASK1 $(5 \mu \mathrm{m})$ was loaded at a DIV of 3 and sustained for 3 days. Subsequently, western blot assays and immunocytochemistry were performed on the NSCs as described below.

\section{NSC primary culture}

Pregnant ICR mice were killed to obtain cortex NSC primary cultures according to the method described by Gritti et al. ${ }^{17}$ Embryonic pup brains were extracted from the ICR mice (E14) and placed in a Petri dish containing Hank's balanced salt solution (Gibco, Grand Island, NY, USA), and each cortex was dissected and washed 1-2 times with plain Hank's balanced salt solution. To each tube of washed tissue, $5 \mathrm{ml}$ of Hank's balanced salt solution was added and pipetted up and down to dissociate the tissue. The NSCs were seeded in stem cell media with EGF and bFGF supplements and were plated on poly-Dlysine-treated plasticware. Cells were seeded at a low density for imaging $\left(3 \times 10^{5}\right.$ cells per $35 \mathrm{~mm}$ dish with coverslip; $2 \times 10^{5}$ cells per well of six-well plate) or on a plate at a high density for biochemistry $\left(3 \times 10^{6}\right.$ cells per well of six-well plate). The cells were cultured for 3 days and were then treated with suitable drugs for each group. Three days after treatment, the NSCs were used for the experiment. Cultures were maintained in a humidified atmosphere of $95 \%$ air and $5 \% \mathrm{CO}_{2}$ at $37^{\circ} \mathrm{C}$.

\section{Western blot analysis}

Western blots were performed to elucidate the molecular mechanism of ASK1 on the differentiation of NSCs. NSCs from each group were cultured until 7 DIV, washed with phosphate-buffered saline, and then collected by scraping the neurospheres from the plate. The collected NSCs were homogenized in lysis buffer (1\% Triton X-100, 0.5\% NP-40, $150 \mathrm{~mm} \mathrm{NaCl}, 10 \mathrm{~mm}$ Tris ( $\mathrm{pH} 7.4$ ), $1 \mathrm{~mm}$ ethylenediaminetetraacetic acid, $1 \mathrm{~mm}$ EGTA pH $8.0,0.2 \mathrm{~mm}$ sodium orthovanadate, $0.2 \mathrm{~mm}$ phenylmethylsulfonyl fluoride and protease inhibitor cocktail) and centrifuged (12000 r.p.m. at $4{ }^{\circ} \mathrm{C}$ ) for $15 \mathrm{~min}$. Equal amounts of protein $(20 \mu \mathrm{g})$ from the supernatants were separated on a $10 \%$ acrylamide gel, and the proteins were electrophoretically transferred onto nitrocellulose membranes. After blocking with Tris-buffered saline with Tween-20, the blots were incubated with primary antibody overnight. The following primary antibodies were used: $\beta$-actin (1:2000, Santa Cruz Biotechnology, Santa Cruz, CA, USA), p38 (1:2000, Cell Signaling, Beverly, MA, USA) and ASK1 (1:1000, Santa Cruz Biotechnology). The membranes were then incubated with horseradish peroxidase-conjugated secondary antibodies, the blots were rinsed, and the protein bands were visualized using an enhanced chemiluminescence detection system (Amersham, Piscataway, NJ, USA).

\section{Flow cytometry analysis}

Apoptotic cells were analyzed using the Annexin V-fluorescein isothiocyanate apoptosis detection kit (Sigma, St Louis, MO, USA) according to the manufacturer's protocol. Neural progenitor cells were dissociated by using Accumax cell detachment solution (Millipore, Billerica, MA, USA). After centrifugation, the resulting suspension was pelleted by centrifugation at 3000 r.p.m. for $3 \mathrm{~min}$ at $4{ }^{\circ} \mathrm{C}$ and then incubated on ice with Annexin V-fluorescein isothiocyanate and propidium iodide. The cells were then incubated at room temperature for $15 \mathrm{~min}$ in the dark. A single-cell suspension was then collected and resuspended in $500 \mu \mathrm{l}$ of fluorescence-activated cell sorting buffer and analyzed using flow cytometry (FACSCalibur system, BD Biosciences, San Jose, CA, USA). All fluorescence-activated cell sorting data were analyzed using FlowJo software (TreeStar, Ashland, OR, USA).

\section{Immunocytochemistry}

To confirm the differentiation of NSCs, NSCs after 7 DIV were washed three times with phosphate-buffered saline for immunostaining and were blocked for $30 \mathrm{~min}$. The NSCs were then incubated with 
primary antibodies overnight at $4{ }^{\circ} \mathrm{C}$. The following primary antibodies were used: anti-MAP2 (1:200, Millipore), anti-SOX2 (1:200, Millipore), anti-Ki-67 (1:200, Millipore) and anti-Nestin (1:200, Millipore). After incubating the NSCs with the primary antibodies, the plates were washed three times with phosphate-buffered saline for $5 \mathrm{~min}$ and were incubated with rhodamine- or fluorescein isothiocyanate-conjugated secondary antibodies. The NSCs were then counterstained with 4,6-diamidino-2-phenylindole for $10 \mathrm{~min}$ at room temperature. Immunostained NSCs were visualized using a Carl-Zeiss confocal microscope LSM 700 (Carl Zeiss, Jena, Germany).

For immunohistochemistry, mice were killed at each time point, and brains were post-fixed in PFA and cut into $20-\mu \mathrm{m}$-thick coronal sections. Fluorescence immunostaining using SOX2 or Ki-67 antibodies was performed, and images were acquired using a confocal microscope LSM700 (Carl Zeiss).

\section{Microarray analysis}

Eight hours after cerebral ischemia, the brains of the mice were homogenized with TRIzol reagent (Invitrogen, Carland, CA, USA) according to the manufacturer's recommendations. In addition, Agilent's Low RNA Input Linear Amplification kit (Agilent Techno-

logy, Santa Clara, CA, USA) was used, and double-stranded DNA was transcribed by adding the transcription master mix (4X transcription buffer, $0.1 \mathrm{M}$ DTT, NTP mix, 50\% PEG, RNase-out, inorganic pyrophosphate, T7-RNA polymerase and cyanine 3/5-CTP) to the double-stranded DNA reaction samples and incubating at $40{ }^{\circ} \mathrm{C}$ for $2 \mathrm{~h}$. After testing the efficiency of labeling, the fragmented cRNA was pipetted onto a Whole Human Genome Microarray Kit $(4 \times 44 \mathrm{~K}$; Agilent Technology), and the hybridized microarrays were washed following the manufacturer's protocol. Using Agilent's DNA microarray scanner, the hybridized images were scanned and quantified using Feature Extraction (Agilent Technology) and GeneSpringGX7.3 (Agilent Technology) software, and all data were normalized and genes of interest were selected by the fold change.

\section{Data analysis}

Statistical comparisons were performed by an independent $t$-test for two groups or an analysis of variance using the SPSS (Statistical Package for the Social Sciences, SPSS 18.0, IBM Corp, New York, NY, USA) software program. The values are expressed as the mean \pm s.e.m. of 3-6 experiments. Differences were considered significant at $P<0.01$ and 0.05

\section{RESULTS}

\section{Cerebral ischemic damage-induced cell death and} endogenous NSCs

Western blot analysis of ischemic stroke-injured mouse brains at each time point showed that ASK1 protein levels were maximized at $8 \mathrm{~h}$ and maintained these levels for 3 days. From day 1, the amounts of ASK1 gradually decreased by 3 and 7 days, but the protein levels were higher than that of the control (Figure 1a). At day 7, SOX2-positive cells were detected in cells that were aligned with the SVZ (Figure 1b). The SOX2 protein, which is a transcription factor, is essential for maintaining the self-renewal, or pluripotency, of undifferentiated, embryonic stem cells.

Eight hours after cerebral ischemia in mice, a significant number of genes were regulated in the ischemic striatum

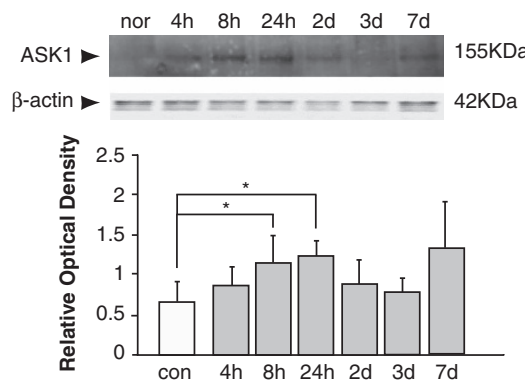

b

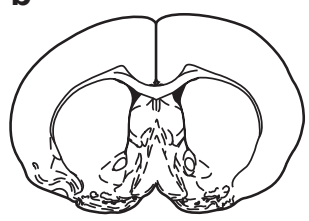

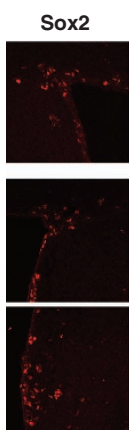

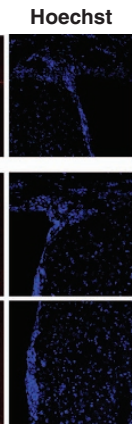

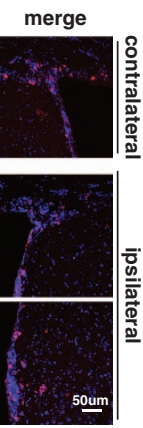

C

\begin{tabular}{|c|c|c|c|c|}
\hline Functional Classification & Gene Symbol & Gene Name & GeneBank Number & Fold Change \\
\hline \multirow{15}{*}{ Inflammation } & $\mathrm{col} 2$ & chemokine (C-C motif) lizand 22 & NM 011333 & 244,98 \\
\hline & $\mathrm{Col} 4$ & chemokine (C-C motif) ligand 4 & NM 013652 & 86.05 \\
\hline & Imb & interleukin I beta & NM 028361 & 69.25 \\
\hline & Cal4 & CDI4 antigen & NM 009841 & 4245 \\
\hline & Cxcl10 & chemokine (C-X-C motif ligand 10 & NM_021274 & 27.00 \\
\hline & Cxels & cherrokine (C-X-C motif ) lieand5 5 & NM_QX91414 & 6.73 \\
\hline & cols & chemokine (C-C motif) ligand 5 & NM 013653 & 6.30 \\
\hline & IIAra & interleukin 4 rececptor, alpha & NM 001008700 & 5.50 \\
\hline & Cell2 & chemokine (C-C motif) ligand 12 & NM 011331 & 5.26 \\
\hline & Cel24 & chemokine (C.C motif) ligand 24 & NM_019577 & 5.25 \\
\hline & 1133 & inmerlecukin 33 & NM_001164724 & 3.09 \\
\hline & $\mathrm{Cd} 24 \mathrm{~s}$ & $\mathrm{CD} 24$ antigen & NM 009816 & 2.89 \\
\hline & Cod4 & CDM4 antigen & NM_009851 & 2.72 \\
\hline & $\mathrm{Cel}_{25}$ & chemekine (C-C moin) ligand 25 & NR 033527 & 2.16 \\
\hline & Cxcll1 & chemokine (C-X-C motiff ligand II & NM 019444 & 204 \\
\hline
\end{tabular}

Figure 1 Upregulation of the levels of apoptosis signal-regulating kinase 1 (ASK1) and endogenous neural stem cells after cerebral ischemia. (a) Western blotting showed that the relative level of ASK1 was elevated from 8 to $24 \mathrm{~h}$ after cerebral ischemia in ischemic lesions. $\beta$-Actin was used as an internal control (mean \pm s.d., $n=3-4$ ). (b) Following cerebral ischemia for 7 days, SOX2-positive cells (red) were observed in the subventricular zone of the contralateral and ipsilateral hemispheres. Hoechst 33258 was used as a counterstain (blue). (c) The tables show significantly upregulated genes that were categorized as being related to inflammation or neurogenesis by assessing the microarrays. Scale bar $=50 \mu \mathrm{m} ;{ }^{*} P<0.1$ compared with normal group. 
(Figure 1c). The Affymetrix scatter plot shows the signal intensity of upregulated genes in the ischemic group compared with that of the normal group, specifically in the categories of inflammation and neurogenesis. On the basis of the table, the following elevated genes were involved in inflammation and neurogenesis: inflammation-CCL2, CCL4 and interleukin1 $\beta$; neurogenesis-Ascl2, Egr2 and P2ry2.

\section{Downregulation of ASK1 influenced neurosphere formation and cell death}

It is well known that neuro-inflammation is induced after an ischemic stroke; therefore, we evaluated this process using our microarray data (Figure 1c). To investigate the role of ASK1 in

endogenous proliferation and NSC differentiation, NSCs from a mouse cortex were cultured, and differentiation was induced by lipopolysaccharide (LPS; $10 \mathrm{ng} \mathrm{ml}^{-1}$ ) treatment. It was confirmed that the treatment of NSCs with ASK1-siRNAs decreased ASK1 protein expression, whereas the ASK1 protein level was not significantly different in the control group, which had been treated with siPORT NeoFX as a vehicle and a scrambled siRNA as a negative control (Figure 2a). To confirm gene silencing, a siRNA against glyceraldehyde 3-phosphate dehydrogenase was used as a positive control.

Bright-field microscope images showed that LPS treatment of NSCs decreased the size of the neurosphere compared with the control group, and the neurospheres of the LPS-treated

b
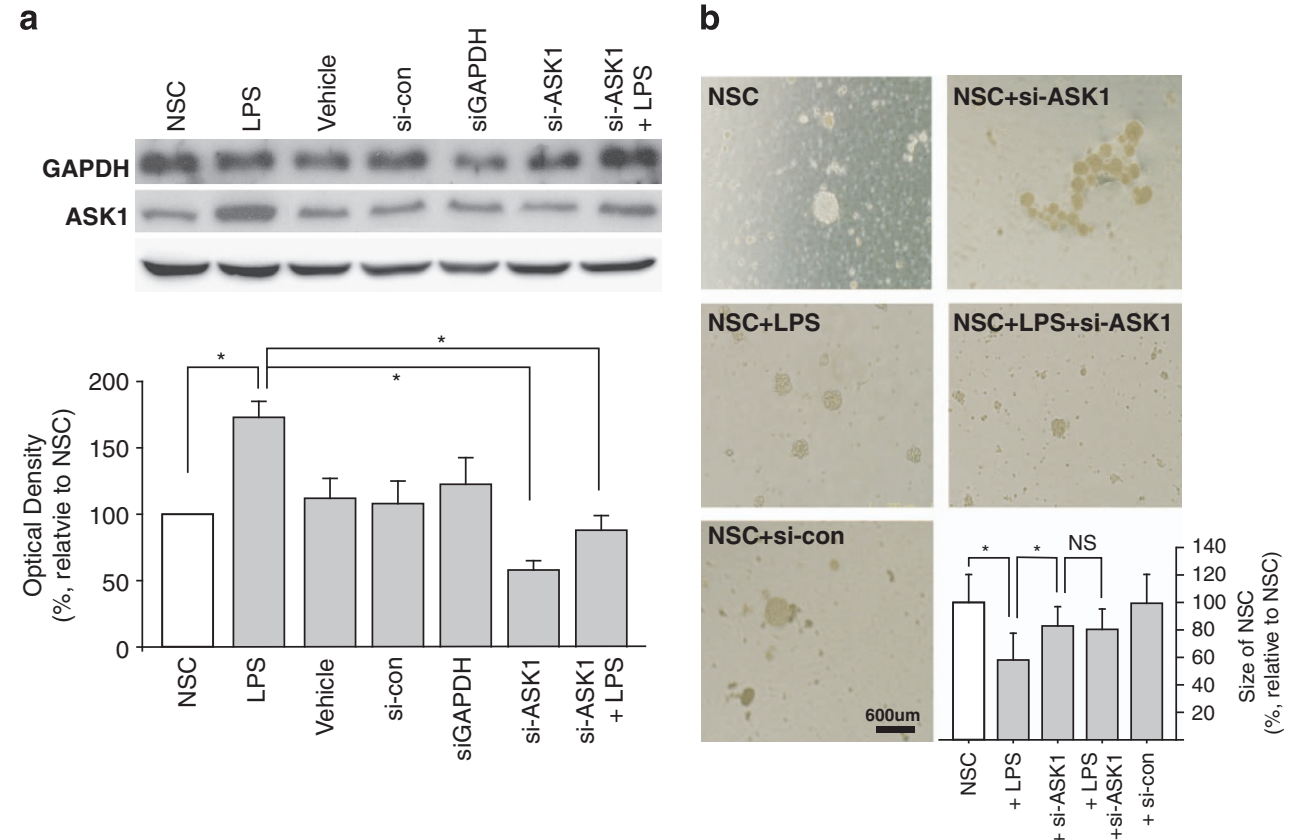

c
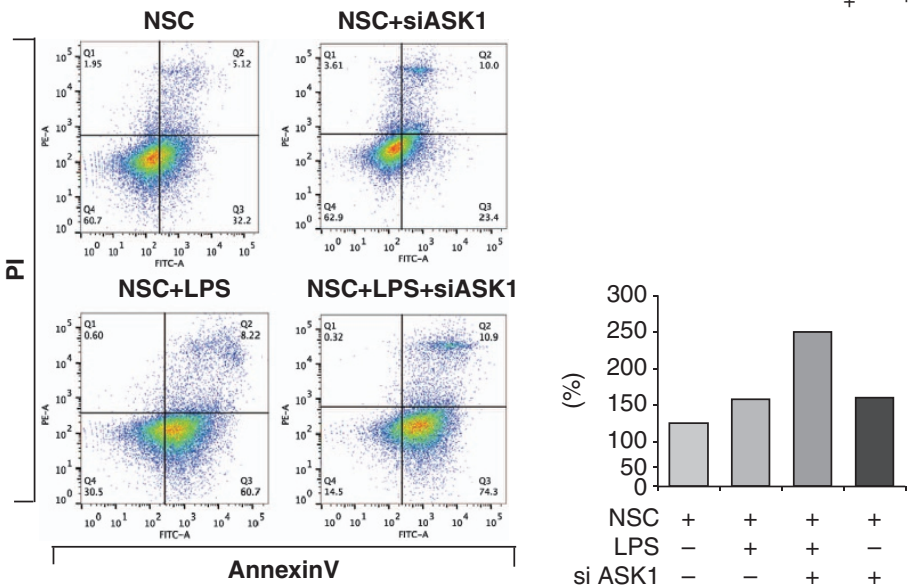

Figure 2 Relationship between cell death and differentiation in neural stem cells. The neurosphere size was measured by bright field microscopy, and cell death was measured by FACS analysis. (a) GAPDH gene silencing was used as a positive control and reduced the level of GAPDH protein. Downregulation was confirmed by western blot analysis (upper). Treatment with the ASK1-siRNA resulted in decreased ASK1 protein levels in NSCs (lower). (b) Neurosphere sizes were maintained more in the ASK1-siRNA treatment group than in the control group. (c) FACS analysis data show that the percentage of dead cells was lower in the ASK1-siRNA treatment group than in the control group after LPS treatment. ${ }^{*} P<0.1$. ASK1, apoptosis signal-regulating kinase 1 ; FACS, fluorescence-activated cell sorting; GAPDH, glyceraldehyde 3-phosphate dehydrogenase; LPS, lipopolysaccharide; NS, not significant; NSC, neural stem cell; PI, propidium iodide; siRNA, small interfering RNA. 
NSCs decreased to below 50\% that of the control group (Figure $2 \mathrm{~b}$ ). Neurosphere size data suggested the possibility that suppression of the ASK1 gene allowed a NSC to maintain its neurosphere in spite of the inflammation condition. To investigate whether the suppression of the ASK1 gene was linked to cell apoptosis, flow cytometry analysis was performed at 6 DIV for each condition (Figure 2c). The flow cytometer reads 50000 cells per sample, and single cells were gated. The propidium iodide and fluorescein isothiocyanate signals were read, and each signal-positive cell was presented as a percentage on the dot plot. Apoptotic cell death was compared between treated groups by counting the Annexin V-positive cells on the dot plot. The quantitative graph showed that ASK1 treatment significantly decreased the number of dead cells compared with that of the negative control and increased the mean diameter of the neurospheres at the same concentration of seeding NSCs $\left(3.0 \times 10^{4}\right.$ cells ml $\left.{ }^{-1}\right)$.

\section{ASK1 is linked to the differentiation of NSCs under LPS-induced inflammation}

Double fluorescent immunostaining results showed that the level of DCX-positive cells, which is a NSC marker, was higher in the LPS-treated than in the control group. In addition, differentiation to mature neurons was detected by MAP2 (a marker of a mature neuron), which was increased by LPS (Figure 3). In the immunocytochemistry analysis, it was difficult to evaluate the changing pattern of DCX- and MAP2-positive cells between treated groups. Therefore, western blotting was performed to confirm the difference in the proliferation and differentiation of NSCs.

In the LPS-treated group, more DCX-positive, NSCs and MAP2-positive, mature neuronal cells were detected than in the control group (Figure 4a). This result indicated that LPS led to stem cell differentiation and proliferation. The results of the western blot analysis after ASK1-siRNA treatment demonstrated that ASK1-siRNA treatment significantly decreased DCX and MAP2 protein expression to normal levels. In contrast to the MAP2 level, the DCX protein level did not undergo a dramatic increase after LPS treatment.

We hypothesize that LPS may have a more significant role in the differentiation rather than proliferation of NSCs. In addition, the inflammatory condition may initiate differentiation by regulating the ASK1 level and its downstream cascades. Based on previous microarray data, ASK1 is the link between the MAPKs-p38 MAPK pathway and neurogenesis. Treatment with ASK1-siRNA seems to modulate the p38 MAPK pathway
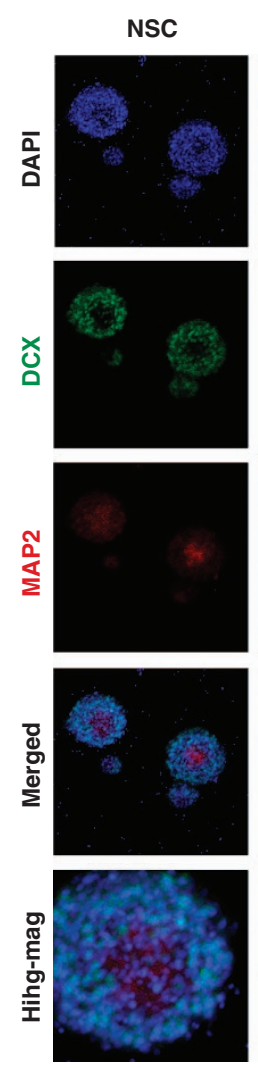

+LPS
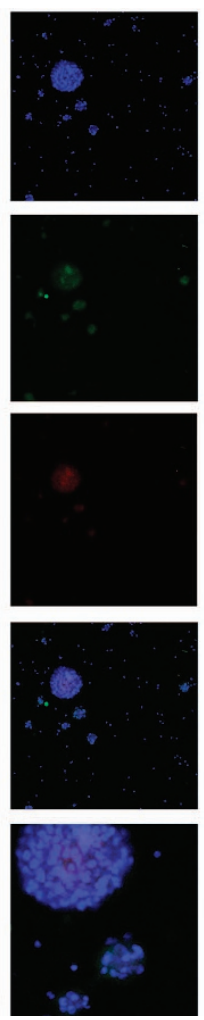

+Vehicle
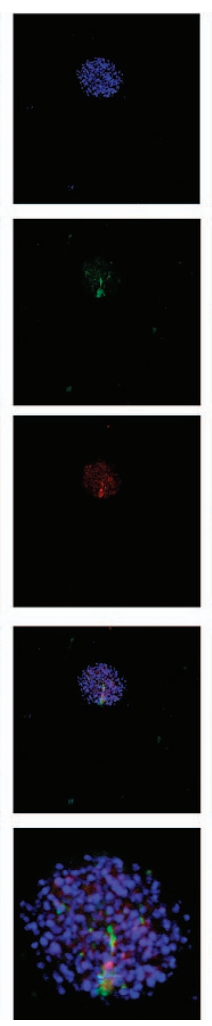

+SIASK1
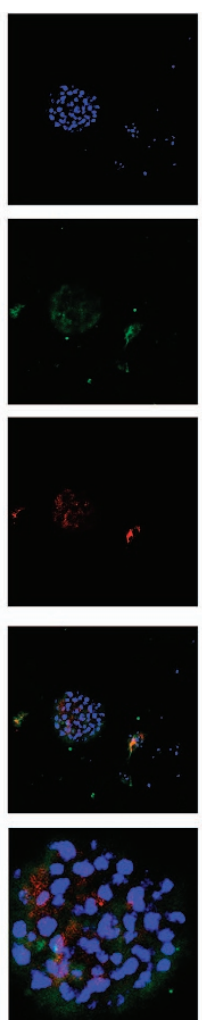

+LPS + si ASK1
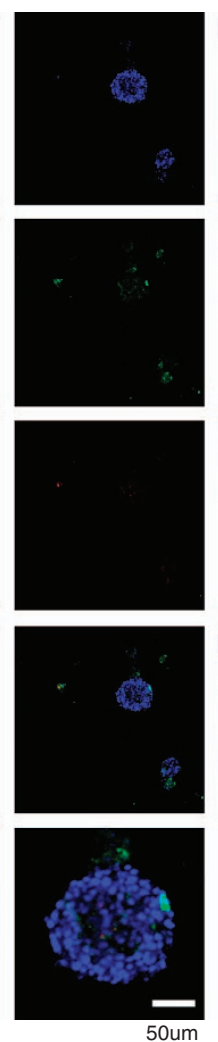

(-) Ab
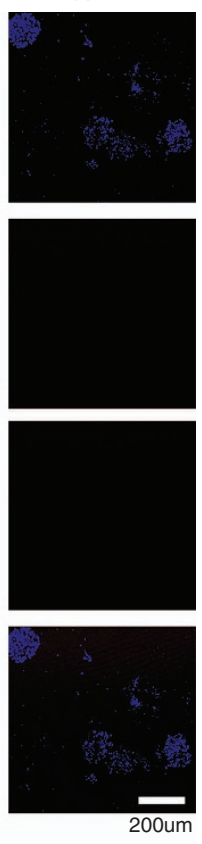

Figure 3 Photomicrographs of neural stem cells (NSCs) under apoptosis signal-regulating kinase 1 (ASK1) suppression conditions. Immunofluorescence images of a neural stem cell (DCX, green) and mature neuron (MAP2, red). The number of MAP2-positive cells increased after ASK1-small interfering RNA (siRNA) treatment in the lipopolysaccharide (LPS)-treated group compared with the LPS-only treatment group. The lane furthest to the right presents results without the primary antibody as a negative control. The bottom row shows the images after higher magnification. 

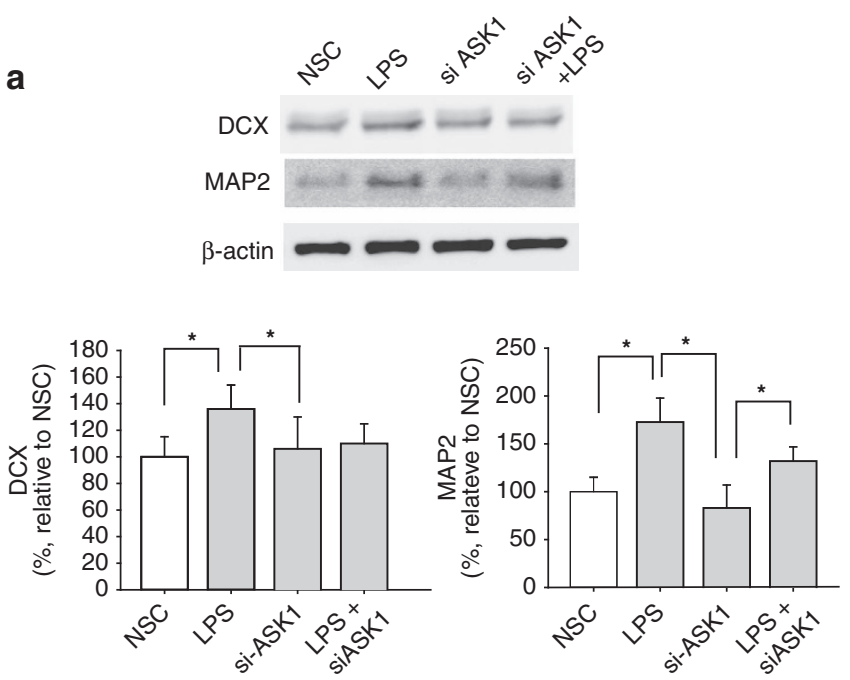

b
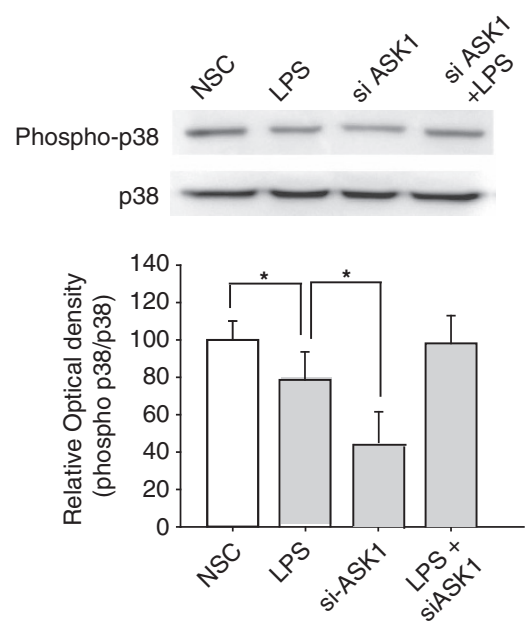

Figure 4 Effect of apoptosis signal-regulating kinase 1 (ASK1) on neuronal differentiation through the p38 mitogen-activated protein kinase (MAPK) pathway. (a) DCX and MAP2 expression increased in the ASK1-small interfering RNA (siRNA) treatment group compared with the control group. (b) Phosphor-p38 expression decreased more in the ASK1-siRNA and the ASK1-siRNA with lipopolysaccharide (LPS)-treatment groups than in the LPS-only treatment group. ${ }^{*} P<0.1$.

and govern cell death and cell fate, thereby regulating the differentiation of NSCs (Figure 4b).

\section{DISCUSSION}

NSCs can possibly self-renew and differentiate into neurons and other glial cells. We aimed to investigate the regulatory effect of ASK1 on NSC differentiation and cell fate determination under controlled ASK1 downregulation. Our present study showed the following: (1) inflammatory conditions such as LPS treatment led NSCs to differentiate and caused cell death. (2) Downregulated ASK1 could not stimulate NSC differentiation. (3) After inducing inflammatory conditions and augmenting ASK1, moderate levels of ASK1 may be effective in differentiating NSCs and in preventing cell death.
Three populations of precursors, including adult NSCs, are found in the SVZ, which is adjacent to a layer of ependymal cells that line the lateral ventricle wall. These NSCs are known to be relatively quiescent cells that express embryonic radial precursors and the astrocyte protein GFAP. Initial studies established the lineage and anatomical relationships of these populations, ${ }^{18}$ but recent studies have expanded our NSC knowledge to the adult SVZ niche. ${ }^{19-21}$ Here, we suggest that ASK1, a MAP3K5, induces neuronal cell death in infarcted areas and neuronal differentiation of NSCs in the SVZ. Similarly, microarray data demonstrate that interleukin-1 $\beta$, a pro-inflammatory factor, was greatly increased, meaning that inflammation was triggered by ischemic insults.

Our previous study also reported that LPS introduced into the corpus callosum of mice increases interleukin- $1 \beta$ levels. ${ }^{22}$ We performed experiments under LPS-induced inflammatory conditions. Under inflammation conditions that were induced by cerebral ischemia, ASK1 levels remained significantly but slightly increased compared with the control group at day 7 . Correspondingly, SOX2-positive cells were detected in the SVZ at day 7 (data not shown), and it could be speculated that moderate amounts of ASK1 were stimulated. ${ }^{23,24}$ Among the neural subtype cell lineages expressing neural SOX2, MAP2 and GFAP, the cells located in the SVZ expressed SOX2. SOX2positive cells can produce self-renewal cells and differentiate to neuronal cells; therefore, they can be used as a stem cell marker. ${ }^{25}$ It has been reported that proliferation of SOX2-positive NSCs can produce neural precursors and maintain NSCs. ${ }^{25}$

We investigated the role of ASK1 in the survival, proliferation and differentiation of central nervous system stem cells by using NSCs. Our data showed that after LPS induction, NSCs that had been treated with ASK1-siRNA had a significantly higher number of cells displaying neuronal features compared with those of control cultures. We also investigated the in vitro effects of LPS and LPS with ASK1-siRNA on NSCs during proliferation and differentiation. The proliferation rate was evaluated through neurosphere formation assays. The size of the neurosphere reflects the state of proliferation or differentiation, and LPS caused the size of the neurosphere to decrease. One explanation is that the NSCs formed a neurosphere that began to differentiate. However, ASK1-siRNA treatment increased the size of the neurosphere. These results are consistent with those of a previous report that found that ASK1 stimulated neuronal differentiation in adult hippocampus-derived progenitor cells. ${ }^{26}$ Until now, the major role of ASK1 was thought to be as an initiating factor for the MAPK cascade and in regulating apoptosis signals. ${ }^{12,27}$

To determine the sub-population ratio of neuronal and glial cells that respond to ASK1 levels, it would be necessary to differentiate between those cells and analyze their responses to ASK1. The previous study by Takeda et al. ${ }^{10}$ showed that the survival and neuronal differentiation of undifferentiated PC12 cells were induced by ASK1, and this induction was dependent on activation of the p38 MAPK. Owing to the different properties of progenitor cells in the peripheral and central nervous systems, neuronal differentiation via the ASK1-p38 MAPK pathway could 
be restricted to cells of the peripheral nervous system. Nonetheless, another previous report demonstrated that ASK1 depletion increased GFAP promoter activity after treatment with SB $203580 .^{26}$ In addition, the inhibition of glial cell differentiation is mediated by the p38 MAPK. ${ }^{26}$ Therefore, it remains to be investigated whether neuronal fate is induced in pre-differentiated precursors or only stimulated in neurons. It seems more likely that glial differentiation in NSCs or early glial precursors is prevented by ASK1.

Severe cerebral ischemic damage raises the possibility of NSCs remaining in a state of quiescence, whereas mild hypoxia brings about NSC proliferation and neuronal and oligodendroglial differentiation. ${ }^{28}$ Therefore, modulating the severity of ischemic damage could provide an important advantage and a useful tool for NSC-mediated therapy of ischemic stroke, and alleviating the high ASK1 levels that occur because of brain damage could aid in modulating this damage. Moderate amounts of ASK1 may promote the differentiation of endogenous stem cells into neurons, whereas downregulated ASK1 levels could simultaneously prevent cell death within damaged lesions. Therefore, regulating ASK1 to a moderate level in an ischemic lesion after a severe injury may result in a more favorable microenvironment. An increased understanding of ASK1 signaling during the differentiation of NSCs may be potentially valuable for future, cell-based therapeutic strategies for neuronal diseases.

\section{CONFLICT OF INTEREST}

The authors declare no conflict of interest.

\section{ACKNOWLEDGEMENTS}

This work was supported by a National Research Foundation of Korea (NRF) grant that was funded by the Korean government (MEST; 2012-0005440). This work was supported by the Brain Korea 21 Project for Medical Science, Yonsei University.

1 Abe K, Aoki M, Kawagoe J, Yoshida T, Hattori A, Kogure K et al. Ischemic delayed neuronal death. A mitochondrial hypothesis. Stroke 1995; 26: 1478-1489.

2 Lindvall O, Kokaia Z. Stem cells in human neurodegenerative disorderstime for clinical translation? J Clin Invest 2010; 120: 29-40.

3 Reynolds BA, Weiss S. Generation of neurons and astrocytes from isolated cells of the adult mammalian central nervous system. Science 1992; 255: 1707-1710

4 Reynolds GP. Developments in the drug treatment of schizophrenia. Trends in pharmacol sci 1992; 13: 116-121.

5 Fuentealba LC, Obernier K, Alvarez-Buylla A. Adult neural stem cells bridge their niche. Cell Stem Cell 2012; 10: 698-708.

6 Graf T, Enver T. Forcing cells to change lineages. Nature 2009; 462: 587-594.

7 Olanow CW, Kieburtz K, Schapira AH. Why have we failed to achieve neuroprotection in Parkinson's disease? Annals of neurology 2008; 64 (Suppl 2), S101-S110

8 Pluchino S, Zanotti L, Rossi B, Brambilla E, Ottoboni L, Salani G et al. Neurosphere-derived multipotent precursors promote neuroprotection by an immunomodulatory mechanism. Nature 2005; 436: 266-271.
9 Lau D, Ogbogu U, Taylor B, Stafinski T, Menon D, Caulfield T. Stem cell clinics online: the direct-to-consumer portrayal of stem cell medicine. Cell Stem Cell 2008; 3: 591-594.

10 Takeda K, Hatai T, Hamazaki TS, Nishitoh H, Saitoh M, Ichijo H. Apoptosis signal-regulating kinase 1 (ASK1) induces neuronal differentiation and survival of PC12 cells. J Biol Chem 2000; 275: 9805-9813.

11 Ichijo H, Nishida E, Irie K, ten Dijke P, Saitoh M, Moriguchi T et al. Induction of apoptosis by ASK1, a mammalian MAPKKK that activates SAPK/JNK and p38 signaling pathways. Science 1997; 275: 90-94.

12 Tobiume K, Matsuzawa A, Takahashi T, Nishitoh H, Morita K, Takeda K et al. ASK1 is required for sustained activations of JNK/p38 MAP kinases and apoptosis. EMBO Rep 2001; 2: 222-228.

13 Sayama K, Hanakawa Y, Shirakata Y, Yamasaki K, Sawada Y, Sun L et al. Apoptosis signal-regulating kinase 1 (ASK1) is an intracellular inducer of keratinocyte differentiation. J Biol Chem 2001; 276: 999-1004.

14 Carmichael ST. Cellular and molecular mechanisms of neural repair after stroke: making waves. Ann Neurol 2006; 59: 735-742.

15 Tsai PT, Ohab JJ, Kertesz N, Groszer M, Matter C, Gao J et al. A critical role of erythropoietin receptor in neurogenesis and post-stroke recovery. J Neurosci 2006; 26: 1269-1274.

$16 \mathrm{Kim} \mathrm{HW}$, Cho KJ, Lee SK, Kim GW. Apoptosis signal-regulating kinase 1 (Ask1) targeted small interfering RNA on ischemic neuronal cell death. Brain Res 2011; 1412: 73-78.

17 Gritti A, Parati EA, Cova L, Frolichsthal P, Galli R, Wanke E et al. Multipotential stem cells from the adult mouse brain proliferate and selfrenew in response to basic fibroblast growth factor. J Neurosci 1996; 16: 1091-1100.

18 Kokovay E, Shen Q, Temple S. The incredible elastic brain: how neural stem cells expand our minds. Neuron 2008; 60: 420-429.

19 Mirzadeh Z, Merkle FT, Soriano-Navarro M, Garcia-Verdugo JM, AlvarezBuylla A. Neural stem cells confer unique pinwheel architecture to the ventricular surface in neurogenic regions of the adult brain. Cell Stem Cell 2008; 3: 265-278.

20 Tavazoie M, Van der Veken L, Silva-Vargas V, Louissaint M, Colonna L, Zaidi B et al. A specialized vascular niche for adult neural stem cells. Cell Stem Cell 2008; 3: 279-288.

21 Shen Q, Wang Y, Kokovay E, Lin G, Chuang SM, Goderie SK et al. Adult SVZ stem cells lie in a vascular niche: a quantitative analysis of niche cellcell interactions. Cell Stem Cell 2008; 3: 289-300.

22 Ahn SK, Hong S, Park YM, Choi JY, Lee WT, Park KA et al. Protective effects of agmatine on lipopolysaccharide-injured microglia and inducible nitric oxide synthase activity. Life Sci 2012; 91: 1345-1350.

23 Yuan T, Liao W, Feng NH, Lou YL, Niu X, Zhang AJ et al. Human induced pluripotent stem cell-derived neural stem cells survive, migrate, differentiate, and improve neurological function in a rat model of middle cerebral artery occlusion. Stem Cell Res Ther 2013; 4: 73.

24 Zhang RL, Chopp M, Roberts C, Jia L, Wei M, Lu M et al. Ascl1 lineage cells contribute to ischemia-induced neurogenesis and oligodendrogenesis. J Cereb Blood Flow Metab 2011; 31: 614-625.

25 Suh H, Consiglio A, Ray J, Sawai T, D'Amour KA, Gage FH. In vivo fate analysis reveals the multipotent and self-renewal capacities of Sox $2+$ neural stem cells in the adult hippocampus. Cell Stem Cell 2007; 1: 515-528.

26 Faigle R, Brederlau A, Elmi M, Arvidsson Y, Hamazaki TS, Uramoto H et al. ASK1 inhibits astroglial development via p38 mitogen-activated protein kinase and promotes neuronal differentiation in adult hippocampusderived progenitor cells. Mol Cell Biol 2004; 24: 280-293.

27 Takeda K, Matsuzawa A, Nishitoh H, Ichijo H. Roles of MAPKKK ASK1 in stress-induced cell death. Cell Struct Funct 2003; 28: 23-29.

28 Santilli G, Lamorte G, Carlessi L, Ferrari D, Rota Nodari L, Binda E et al. Mild hypoxia enhances proliferation and multipotency of human neural stem cells. PLoS One 2010; 5: e8575.

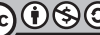

This work is licensed under a Creative Commons Attribution-NonCommercial-ShareAlike 3.0 Unported License. To view a copy of this license, visit http:// creativecommons.org/licenses/by-nc-sa/3.0/ 